\title{
Influence des organes receveurs sur la distribution du carbone photosynthétique entre l'amidon et le saccharose dans la feuille de soja
}

\author{
G Zombré 1, G Viala 1, M Naudy-de-Serres 1, N Gelfi 2, J Calmés 1 \\ 1 Université Paul Sabatier, centre de physiologie végétale, unité de recherche associée au CNRS $n^{\circ} 241$, \\ 118 route de Narbonne, 31062 Toulouse Cedex; \\ 2 INRA, station d'agronomie, centre de recherche de Toulouse, BP 27, 31326 Castanet-Tolosan, France
}

(Reçu le 17 avril 1990; accepté le 16 janvier 1991)

\begin{abstract}
Résumé - L'influence des transferts vers les organes receveurs sur la distribution foliaire du carbone photosynthétique entre l'amidon et le saccharose est étudiée sur des plants de soja (Glycine max, L Merr), variété Kingsoy. Après des incorporations foliaires de ${ }^{14} \mathrm{CO}_{2}$ effectuées in situ, la répartition du radiocarbone entre les 2 glucides et son transfert vers les organes receveurs sont précisés aux cours des h qui suivent l'incorporation. Lorsque les migrations sont importantes ou stimulées par des irrigations fertilisantes, peu de radiocarbone est intégré dans l'amidon. Inversement, si les transferts sont ralentis, davantage de carbone fixé est utilisé pour la synthèse de l'amidon; ce dernier, ainsi que le saccharose, s'accumulent dans la feuille. Ces résultats sont confirmés par des expériences où l'on modifie artificiellement le rapport source/puits. En présence d'une alimentation minérale déséquilibrée, les transferts d'assimilats sont fortement réduits et la teneur foliaire en saccharose augmente; l'activité de la saccharose phosphate synthase diminue; et, malgré une faible teneur en F-2,6-P favorable à la formation du saccharose, davantage de carbone photosynthétique est orienté vers l'amidon.
\end{abstract}

amidon / saccharose / transfert / effet puits / soja

Summary - Influence of sinks on photosynthetic carbon distribution between starch and sucrose in soybean leaf. The influence of translocations towards sinks on photosynthetic carbon distribution between foliar starch and sucrose was studied in soybean plants (Glycine max $L$ Merr), cv Kingsoy. After foliar ${ }^{14} \mathrm{CO}_{2}$ incorporation in situ, the radiocarbon distribution between starch and sucrose and the ${ }^{14} \mathrm{C}$ translocation towards sinks were investigated during the hours following incorporation (fig 1). When the export rate increased or was stimulated by fertilising irrigations, less radiocarbon was incorporated into starch (fig 2). If the translocation decreased, more radiocarbon was used for starch synthesis and the leaf accumulated starch and sucrose (table II). Experiments on source/sink modifications corroborated these data (fig 3). Nutrient solution overloaded with minerals decreased the export rate and increased leaf sucrose content (fig 4); sucrose phosphate synthase activity weakened (table III) and, in spite of small F-2,6-P content facilitating sucrose formation, more photosynthetic carbon was directed towards starch.

starch / sucrose / translocation / sink effect / soybean

\section{INTRODUCTION}

La productivité végétale dépend, pour une large part, du métabolisme foliaire et du transfert des assimilats vers les organes receveurs. Le $\mathrm{CO}_{2}$, fixé par la ribulose bisphosphate carboxylase, est progressivement transformé en trioses- $P$. Ces derniers sont orientés vers la synthèse d'amidon dans le chloroplaste ou du saccharose dans le hyaloplasme. Dans les conditions habituelles, l'amidon s'accumule durant la journée et est utilisé durant la nuit, si bien que la quantité présente dans la feuille au début de la photopériode est faible. Cependant, dans certaines conditions de nutrition minérale (carence en $\mathrm{N}$, Rufty et al, 1988), ou d'éclairement (photopériodes courtes, Carlson et Brun, 1984), la synthèse diurne l'emporte sur l'hydrolyse nocturne et la teneur foliaire devient de plus en plus élevée. Le niveau des réserves amylacées de la feuille traduit souvent une adaptation de la plante à des conditions de développement défavorables et lui permet de mieux résister à certains stress comme, par exemple, le déficit hydrique (Huber et al, 1984; Bensari et al, 1990). 
Le saccharose constitue le métabolite clé : une partie, utilisée sur place, permet la synthèse des divers composés foliaires, l'autre partie est exportée vers les organes receveurs. Des études conduites sur des feuilles isolées ou des disques foliaires ont permis de déterminer les principaux éléments régulateurs de la synthèse du saccharose : le manque de phosphate inorganique ralentit la sortie des trioses- $P$ du chloroplaste (Heldt et al, 1977; Leegood et al, 1988); le caractère limitant de 2 enzymes (fructose-1,6bisphosphatase, saccharose-phosphate synthase) a été précisé (Gerhardt et al, 1987; Stitt et al, 1988; Stitt et Quick, 1989). Toutefois dans une plante entière, un des facteurs prépondérants pourraît être l'effet puits exercé par les organes receveurs sur les transferts foliaires et, par conséquent, sur l'exportation du saccharose hors du hyaloplasme (Foyer, 1987, 1988).

Ce travail étudie la distribution du carbone photosynthétique entre amidon et saccharose dans diverses conditions susceptibles de modifier les transferts foliaires : modifications observées dans des conditions normales de culture ou perturbations engendrées, soit par l'augmentation ou la diminution artificielles des organes receveurs, soit par des alimentations minérales déséquilibrées.

\section{MATÉRIEL ET MÉTHODES}

Du soja (Glycine max, L Merr), variété Kingsoy, inoculé par du Bradyrhizobium japonicum, souche G 49, est cultivé en plein champ, à la station d'agronomie de Toulouse (semis le 21 avril). L'irrigation satisfait sensiblement l'évapotranspiration maximale. Le sol brun, limono-argileux profond, est bien fertilisé en phosphore et potassium.

Pour les expérimentations avec surcharges minérales, du soja non nodulé est cultivé en salle climatisée (héméropériode, $14 \mathrm{~h}$; éclairement $50 \mathrm{~W} . \mathrm{m}^{-2}$ au niveau des feuilles étudiées; température $27{ }^{\circ} \mathrm{C}$ le jour, $20^{\circ} \mathrm{C}$ la nuit; humidité relative $80 \%$ ) sur sable imbibé d'une solution nutritive contenant $\mathrm{KNO}_{3}$ 6 mmol.1-1, $\mathrm{Ca}\left(\mathrm{NO}_{3}\right)_{2} 4 \mathrm{mmol.1}-1, \mathrm{NH}_{4} \mathrm{H}_{2} \mathrm{PO}_{4}$ $1 \mathrm{mmol}^{-1} \mathrm{IgSO}_{4} 2 \mathrm{mmol}^{-1} \mathrm{MgS}^{-1}$, EDTA Fe $70 \mu \mathrm{mol} . \mathrm{I}^{-1}$, $\mathrm{H}_{3} \mathrm{BO}_{3} 50 \mu \mathrm{mol}^{-1}{ }^{-1}, \mathrm{MnCl}_{2}, 9 \mu \mathrm{mol} . \mathrm{I}^{-1}, \mathrm{CuSO}_{4} 0,3$ $\mu \mathrm{mol} . \mathrm{I}^{-1}, \mathrm{ZnSO}_{4} 0,8 \mu \mathrm{mol} . \mathrm{I}^{-1}, \mathrm{Na}_{2} \mathrm{MoO}_{4}$ 0,2 $\mu \mathrm{mol} . \mathrm{I}^{-1}$. Trois semaines après la levée, 5 lots reçoivent pendant $7 \mathrm{j}$ une alimentation modifiée comme suit :

- ajout de $\mathrm{CaCl}_{2} 25 \mathrm{mmol} . \mathrm{I}^{-1}$;

- ajout de $\mathrm{MgCl}_{2} 25$ mmol..1-1;

- ajout de $\mathrm{KCl} 50 \mathrm{mmol} . \mathrm{I}^{-1}$;

- ajout de $\mathrm{KH}_{2} \mathrm{PO}_{4} 100 \mathrm{mmol} . \mathrm{I}^{-1}$;

- alimentation ammoniacale contenant $\mathrm{NH}_{4} \mathrm{Cl} 8$

mmol.. ${ }^{-1}, \quad \mathrm{NH}_{4} \mathrm{H}_{2} \mathrm{PO}_{4} 1 \mathrm{mmol.}{ }^{-1}, \quad\left(\mathrm{NH}_{4}\right)_{2} \mathrm{SO}_{4} \quad 0,5$ mmol.l-1, $\mathrm{K}_{2} \mathrm{HPO}_{4} 3 \mathrm{mmol} . \mathrm{I}^{-1}, \mathrm{CaCl}_{2} 2 \mathrm{mmol}^{-1}$,

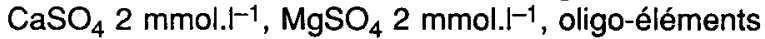
cités ci-dessus.

Les charges en ${ }^{14} \mathrm{CO}_{2}(730 \mathrm{kBq})$, de durée $10 \mathrm{~min}$, sont réalisées sur une feuille adulte trifoliolée maintenue en place sur la plante : le dispositif expérimental a été décrit précédemment (Calmés et Viala, 1987). L'incorporation est répétée sur plusieurs plantes. La première est prélevée 5 min après la fin de la charge; les autres le sont au cours de la journée ou seulement le lendemain. À chaque prélèvement, la feuille traitée est fixée dans l'alcool à $80 \%$. La mesure de la radioactivité des diverses fractions de métabolites et le dosage enzymatique de l'amidon et du saccharose sont exposés par ailleurs (Bensari et al, 1988). Une autre feuille de la même plante, fixée à l'azote liquide, est utilisée pour la mesure de l'activité de la saccharosephosphate synthase (SPS) et pour le dosage du fructose-2,6-bisphosphate (F-2,6-P). L'activité SPS est déterminée par la méthode radiochimique qui mesure la radioactivité du saccharose-phosphate formé à partir d'UDP-14C-glucose (Salerno et al, 1979; Davin, 1987). Le dosage du F-2,6-P repose sur l'activation par ce métabolite de la pyrophosphate-phosphofructokinase de pomme de terre (Van Schaftingen et al, 1982; Zombré et al, 1990).

\section{RÉSULTATS}

\section{Distribution du carbone photosynthétique dans la feuille et transfert vers les organes receveurs}

Des incorporations foliaires de ${ }^{14} \mathrm{CO}_{2}$ de durée 10 min sont réalisées au champ, le matin vers $7 \mathrm{~h}$ (temps universel, TU) au moment du remplissage des graines (stade $R_{6}$ de Fehr et al, 1971) et les prélèvements sont échelonnés toutes les $2 \mathrm{~h}$ au cours de la journée. Le premier est effectué 5 min après la fin de la charge; un dernier prélèvement est réalisé le lendemain matin, $24 \mathrm{~h}$ après l'incorporation.

Les glucides hydrosolubles et l'amidon renferment la majeure partie du radiocarbone foliaire (fig 1); le reste correspond aux acides organiques et aux acides aminés libres et protéiques. Au cours de la journée la diminution de la radioactivité foliaire totale coïncide avec celle des glucides hydrosolubles. Dans cette fraction, le marquage du saccharose prédomine largement durant les premières $h$ qui suivent l'incorporation (tableau I); par la suite, cette prédominance diminue tandis que la radioactivité du glucose et du fructose devient relativement importante. Les 2 oses, en effet, ne sont pas comme le saccha- 


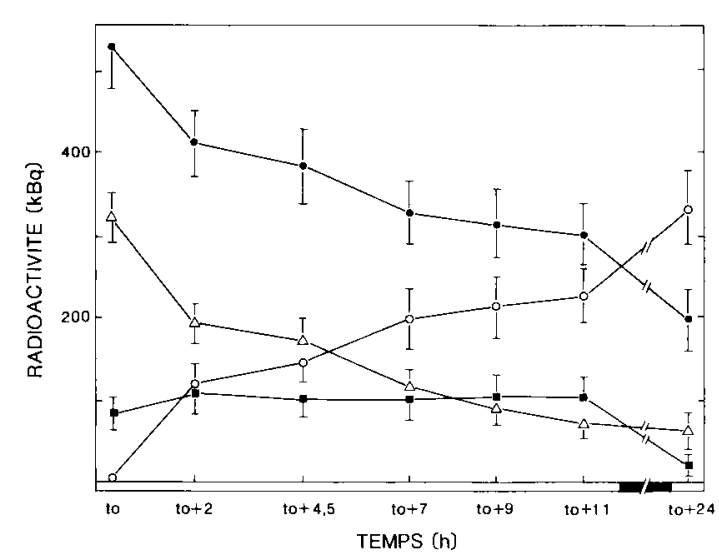

Fig 1. Évolution de la radioactivité au cours des 24 h qui suivent une incorporation de ${ }^{14} \mathrm{CO}_{2}$. Cette dernière est effectuée au champ sur des feuilles in situ, à l'époque du remplissage des graines (stade $R_{6}$ ). Le temps $t_{0}(7 \mathrm{~h} \mathrm{TU}$ ) correspond au premier prélèvement réalisé après $10 \mathrm{~min}$ de charge et $5 \mathrm{~min}$ de chasse. L'alternance lumière-obscurité est indiquée en abscisse par un rectangle blanc ou noir. - , Radioactivité totale du limbe; $\Delta$, glucides hydrosolubles foliaires; $\mathbf{\square}$, amidon foliaire; $O$, radioactivité exportée. Moyenne de 4 échantillons \pm SD.

Tableau I. Distribution (en \%) de la radioactivité entre les principaux glucides hydrosolubles de la feuille, immédiatement après une charge en ${ }^{14} \mathrm{CO}_{2}$ de durée 10 $\min , 2,8$ et 24 h plus tard.

\begin{tabular}{lrrrr}
$\begin{array}{l}\text { Glucide } \\
\text { considéré }\end{array}$ & Oh & $2 h$ & $8 h$ & $24 h$ \\
\hline $\begin{array}{l}\text { Stachyose } \\
\text { Mélibiose }\end{array}$ & 1,9 & 4,8 & 6,8 & 8,1 \\
+ maltose & 5,0 & 8,4 & 9,0 & 9,7 \\
$\begin{array}{l}\text { Saccharose } \\
\text { Galactose }\end{array}$ & 80,1 & 53,6 & 34,5 & 26,9 \\
$\begin{array}{l}\text { Glucose } \\
\text { Fructose }\end{array}$ & 6,7 & 7,8 & 4,3 & 5,4 \\
& 6,0 & 12,5 & 19,8 & 22,2 \\
& & 12,9 & 25,6 & 27,6 \\
\hline
\end{tabular}

rose, transférés vers les organes puits. En revanche la radioactivité de l'amidon se stabilise durant la journée; au cours de la nuit, il est hydrolysé et mobilisé au profit des organes receveurs.

\section{Variations dans la distribution suivant les plantes et l'heure de la journée}

Dans une parcelle correspondant à des conditions culturales normales, est testée l'homogénéité de comportement des plantes au cours de la journée. Pour cela des incorporations foliaires de ${ }^{14} \mathrm{CO}_{2}$ sont réalisées respectivement à $7 \mathrm{~h}, 11 \mathrm{~h}$ et $16 \mathrm{~h}$ (TU) au moment de la floraison (stade $R_{1}$ ) et lors du remplissage des graines (stade $R_{6}$ ). La quantité de radiocarbone transférée vers les organes puits est déterminée $2 \mathrm{~h}$ plus tard. Étant données les migrations rapides du saccharose, l'ensemble de la plante (folioles, pétioles, tige, gousses, racines) est analysé. Le tableau II indique le marquage des divers composés (amidon, composés azotés, acides organiques, glucides hydrosolubles) de la feuille traitée et la radioactivité exportée. Cette dernière, regroupée sous la dénomination "composés exportés", correspond à des glucides pour plus de $95 \%$. L'importance des migrations varie beaucoup d'une plante à l'autre : plutôt que de présenter les valeurs moyennes, il semble intéressant de réunir les plants de soja en 2 ensembles, suivant que leurs transferts sont plus ou moins élevés. Ainsi, les résultats obtenus sont répartis sur 2 colonnes dont la première correspond aux plantes à transferts importants (tableau II); dans ce cas les valeurs du rapport composés exportés/glucides hydrosolubles foliaires sont toujours plus grandes que celles de la $2^{\mathbf{e}}$ colonne. Par contre la quantité de ${ }^{14} \mathrm{C}$ incorporée dans l'amidon est toujours plus élevée lorsque les migrations de photosynthétats sont faibles. Ceci s'observe tout au long de la journée (matin, midi et soir) et aux 2 stades étudiés : floraison et remplissage des graines.

\section{Influence d'irrigations fertilisantes}

Les irrigations fertilisantes, qui généralement améliorent les rendements, sont susceptibles de modifier les transferts d'assimilats foliaires. Les cultures traitées reçoivent $100 \mathrm{~kg} \mathrm{ha}^{-1}$ d'azote sous forme d'urée ajoutée à l'eau d'arrosage : ils sont répartis en 2 interventions aux stades $R_{3}$ et $R_{4}$. Les cultures témoins bénéficient uniquement d'apport d'eau. Pendant le remplissage des graines (stade $R_{6}$ ), après des incorporations de ${ }^{14} \mathrm{CO}_{2}$ de durée $10 \mathrm{~min}$ réalisées à $7 \mathrm{~h}$ (TU), le devenir du radiocarbone foliaire et sa distribution entre l'amidon et le saccharose sont suivis au cours de la journée jusqu'au lendemain matin $7 \mathrm{~h}$ (fig $2 \mathrm{~A}$ ). Durant ces $24 \mathrm{~h}$, la radioactivité foliaire diminue plus vite dans les feuilles des plantes traitées; cette diminution traduit des transferts d'assimilats plus rapides. Corrélativement, la répartition du radiocarbone est modifiée: moins de ${ }^{14} \mathrm{C}$ dans l'amidon et davantage dans les glucides hydrosolubles.

On retrouve les mêmes résultats lorsque les incorporations de ${ }^{14} \mathrm{CO}_{2}$ sont réalisées à $12 \mathrm{~h}$, au début de l'après-midi : la part de radiocarbone orienté vers l'amidon est alors plus faible (fig 2 B). 
Tableau II. Distribution du radiocarbone $2 \mathrm{~h}$ après des incorporations de ${ }^{14} \mathrm{CO}_{2}$ de durée $15 \mathrm{~min}$, effectuées respectivement à 7,11 et $16 \mathrm{~h}$ (TU), au début de la floraison et durant le remplissage des graines. La quantité de ${ }^{14} \mathrm{C}$ retrouvée dans l'ensemble de la plante a été ramenée à $1000 \mathrm{kBq}$. Les pieds où les transferts vers les organes puits sont élevés sont regroupés dans les colonnes I, ceux où les transferts sont faibles dans les colonnes II. Moyenne de 3 échantillons; écart type des mesures voisin de $5 \%$ des valeurs moyennes.

\begin{tabular}{|c|c|c|c|c|c|c|}
\hline \multirow{2}{*}{$\begin{array}{l}\text { Composés } \\
\text { dont la radioactivité } \\
\text { a été mesurée }\end{array}$} & \multicolumn{2}{|c|}{ Matin } & \multicolumn{2}{|c|}{ Midi } & \multicolumn{2}{|c|}{ Soir } \\
\hline & 1 & II & 1 & II & 1 & II \\
\hline
\end{tabular}

\section{Floraison}

Composés foliaires

$\begin{array}{lllllll}\text { amidon } & 146 & 239 & 49 & 61 & 62 & 68 \\ \text { composés azotés } & 175 & 159 & 117 & 111 & 118 & 127 \\ \text { acides organiques } & 159 & 80 & 119 & 152 & 140 & 114 \\ \text { glucides hydrosolubles } & 198 & 222 & 312 & 307 & 249 & 316 \\ \text { Composés exportés } & 322 & 300 & 403 & 369 & 431 & 375 \\ \text { C exportés/G hydrosolubles } & (1,63) & (1,35) & (1,29) & (1,20) & (1,73) & (1,19)\end{array}$

Remplissage des graines

Composés foliaires

amidon

composés azotés

acides organiques

glucides hydrosolubles

Composés exportés

C exportés/ $G$ hydrosolubles

$\begin{array}{rl}101 & 108 \\ 72 & 54 \\ 39 & 64 \\ 322 & 396 \\ 466 & 378 \\ (1,45) & (0,95)\end{array}$

111
58
57
274
500
$(1,82)$

181
48
78
358
335
$(0,94)$

$\begin{array}{cc}110 & 151 \\ 57 & 72 \\ 103 & 102 \\ 193 & 442 \\ 537 & 233 \\ (2,78) & (0,53)\end{array}$

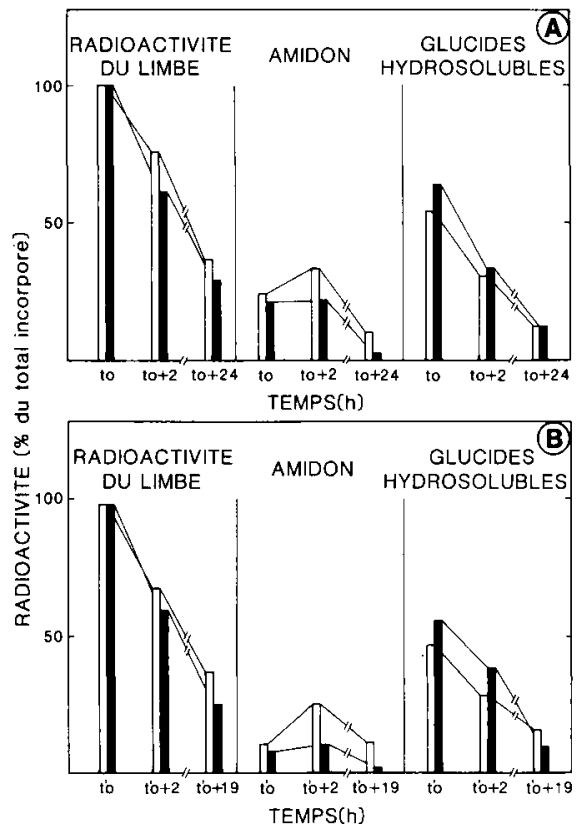

Fig 2. Influence des irrigations fertilisantes sur l'évolution de la radioactivité de l'ensemble du limbe et, en particulier, de l'amidon et des glucides hydrosolubles. Les incorporations de ${ }^{14} \mathrm{CO}_{2}$ sont réalisées au champ sur des feuilles in situ à l'époque du remplissage des graines (stade $R_{6}$ ). $A$, incorporations effectuées le matin à $t_{0}=7 \mathrm{~h}$ (TU); $\mathrm{B}$, incorporations effectuées en milieu de journée à $t^{\prime}=12 \mathrm{~h} ; 0$, témoin; $\mathbf{D}$, irrigations fertilisantes. Moyenne de 3 échantillons.

\section{Effets des modifications du rapport source/puits}

Les relations entre la feuille source et les organes puits ont été modifiées. L'effet puits est augmenté en ne laissant, $24 \mathrm{~h}$ avant l'incorporation, qu'une feuille adulte pour alimenter l'ensemble de la plante. Dans ce cas, moins de ${ }^{14} \mathrm{C}$ est incorporé dans l'amidon et les teneurs foliaires en amidon et saccharose diminuent (fig 3 ). Inversement, l'effet puits est diminué en supprimant, $24 \mathrm{~h}$ auparavant :

- l'apex et les jeunes feuilles en plein développement pour l'expérimentation réalisée à la floraison,

- l'ensemble des gousses, pour l'expérimentation effectuée lors du remplissage des graines. Davantage de ${ }^{14} \mathrm{C}$ est alors incorporé dans l'amidon et les teneurs foliaires en amidon et saccharose augmentent. Les modifications observées sont sensiblement de même importance à la floraison et durant le remplissage des graines; toutefois dans ce dernier cas, les teneurs sont plus élevées. 


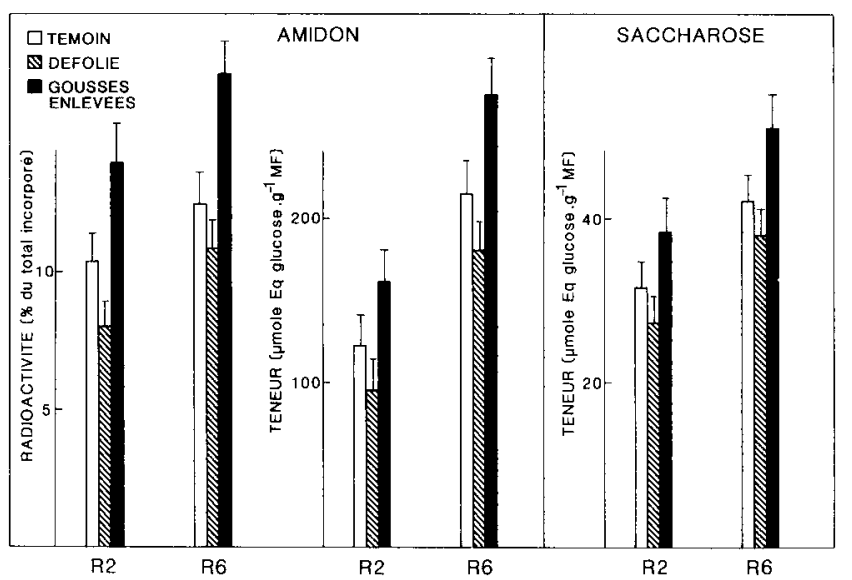

Fig 3. Influence des modifications du rapport source/puits, effectuées $24 \mathrm{~h}$ avant le prélèvement, sur la répartition du carbone photosynthétique entre amidon et saccharose. L'effet puits est augmenté en supprimant toutes les feuilles, sauf la feuille source. L'effet puits est diminué en supprimant l'apex et les jeunes feuilles pour l'expérimentation effectuée à la floraison $\left(R_{2}\right)$, l'ensemble des gousses pour l'expérimentation effectuée lors du remplissage des graines $\left(R_{6}\right)$. Moyenne de 12 échantillons \pm SD.

\section{Effets d'un déséquilibre dans la nutrition minérale sur les transferts}

En soumettant les cultures de soja à des alimentations minérales déséquilibrées, il est possible de ralentir les transferts d'assimilats foliaires et d'étudier l'effet de ce ralentissement sur la répartition du carbone photosynthétique entre amidon et saccharose. Ces conditions n'étant pas réalisables au champ, les essais sont effectués en salle climatisée et 5 types de surcharges minérales sont étudiées.

La figure 4 indique la part de radioactivité transférée $4 \mathrm{~h}$ après des incorporations de ${ }^{14} \mathrm{CO}_{2}$ effectuées in situ. Dans tous les cas de surcharge, les migrations sont ralenties : la proportion de ${ }^{14} \mathrm{C}$ transférée atteint $63 \%$ chez le témoin et seulement $42 \%$ pour $\mathrm{NH}_{4}, 41 \%$ pour $\mathrm{CaCl}_{2}$, $35 \%$ pour $\mathrm{MgCl}_{2}, 28 \%$ pour $\mathrm{KCl}$ et $21 \%$ pour $\mathrm{KH}_{2} \mathrm{PO}_{4}$. En même temps, la synthèse de l'amidon est favorisée : par rapport aux plantes témoins, elle est en moyenne 2 fois plus importante; elle atteint même 3 fois plus dans le cas de surcharge en $\mathrm{KH}_{2} \mathrm{PO}_{4}$. Pour évaluer la synthèse totale en glucides hydrosolubles, constitués essentiellement de saccharose, il faut ajouter à la fraction restant dans la feuille, celle qui a été exportée (fig 4). Le rapport glucides hydrosolubles totaux/amidon, qui est de 7,5 chez le témoin, diminue lorsque les transferts sont perturbés : 4,3 avec $\mathrm{CaCl} ; 3,5$ avec $\mathrm{NH}_{4} ; 3,3$ avec $\mathrm{MgCl}_{2} ; 2,9$ avec $\mathrm{KCl}$ et seulement 1,4 avec $\mathrm{KH}_{2} \mathrm{PO}_{4}$.

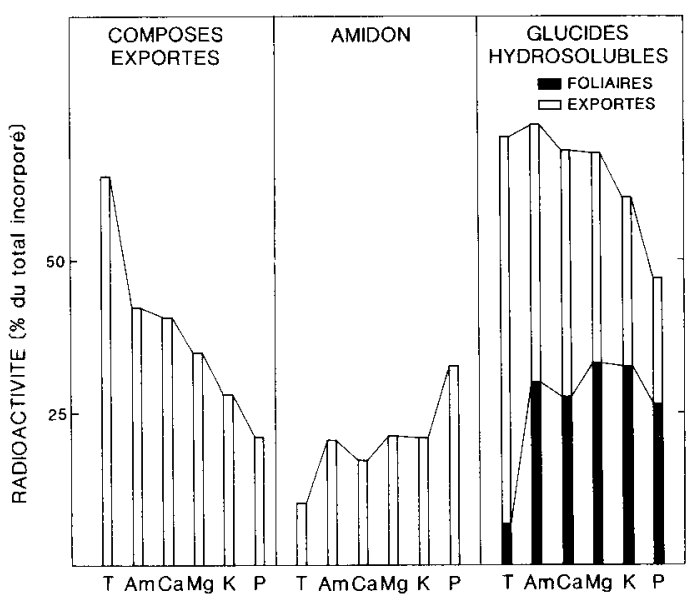

Fig 4. Effet des solutions nutritives surchargées en éléments minéraux sur l'exportation des assimilats et sur la distribution du radiocarbone entre l'amidon et les glucides hydrosolubles foliaires. Les surcharges sont appliquées durant $7 \mathrm{j}$, 3 semaines après la levée. Les prélèvements sont effectués $4 \mathrm{~h}$ après une incorporation foliaire de ${ }^{14} \mathrm{CO}_{2}$. $\mathrm{T}$ : témoin; $\mathrm{Am}$ : nutrition ammoniacale; $\mathrm{Ca}$ : surcharge en $\mathrm{CaCl}_{2}$ 25 mmol..$^{-1} ; \mathrm{Mg}$ : surcharge en $\mathrm{MgCl}_{2} 25 \mathrm{mmol}^{-\mathrm{I}^{-1}} ; \mathrm{K}$ : sucharge en $\mathrm{KCl} 50$ mmol. ${ }^{-1}$; $\mathrm{P}$ : surcharge en $\mathrm{KH}_{2} \mathrm{PO}_{4} 100$ mmol..$^{-1}$. Moyenne de 3 échantillons. Ecart type des mesures voisin de $10 \%$ des valeurs moyennes.

Les surcharges en ions minéraux provoquent une augmentation de la teneur foliaire en amidon (sauf dans le cas de $\mathrm{KCl}$ ) et en saccharose tandis que l'activité de la SPS devient plus faible (tableau III). La teneur en F-2,6-P diminue nettement dans les jeunes limbes en croissance; la diminution est plus limitée dans les feuilles adultes sauf pour les cultures surchargées en $\mathrm{KH}_{2} \mathrm{PO}_{4}$ où elle est importante.

\section{DISCUSSION}

L'incorporation foliaire de ${ }^{14} \mathrm{CO}_{2}$ permet de déterminer les proportions de carbone photosynthétique intégrées dans le saccharose et l'amidon; par voie de conséquence les variations dans les activités de synthèse de ces 2 glucides peuvent être précisées. Pour limiter l'analyse à la feuille qui a reçu l'incorporation, il convient de fixer le matériel végétal avant le début des transferts; sinon il faut prendre en compte le radiocarbone exporté et analyser l'ensemble de la plante. En moyenne, $2 \mathrm{~h}$ après l'incorporation, la quantité de glucides transférée, essentiellement sous forme de saccharose, est plus importante que celle restée dans la feuille (tableau II). Cette méthode basée sur la distribution du radiocarbone a été complétée par l'étude des teneurs foliaires en amidon et saccharose. Si elles ne per- 
Tableau III. Teneurs en amidon et saccharose ( $\mu \mathrm{mol}$ (Eq glucose). $\mathrm{g}^{-1}$ (matière fraîche), activité SPS ( $\mu \mathrm{mol}$ (saccharose formé). $\mathrm{h}^{-1} \cdot \mathrm{g}^{-1}$ (matière fraîche)) dans une feuille trifoliolée adulte. Teneur en F-2,6-P (nmol.g-1 (matière fraîche)) dans une feuille adulte (I) et en début de croissance (II). Prélèvements effectués après $8 \mathrm{~h}$ d'éclairement. Moyenne de 4 échantillons; écart type des mesures voisin de $10 \%$ des valeurs moyennes.

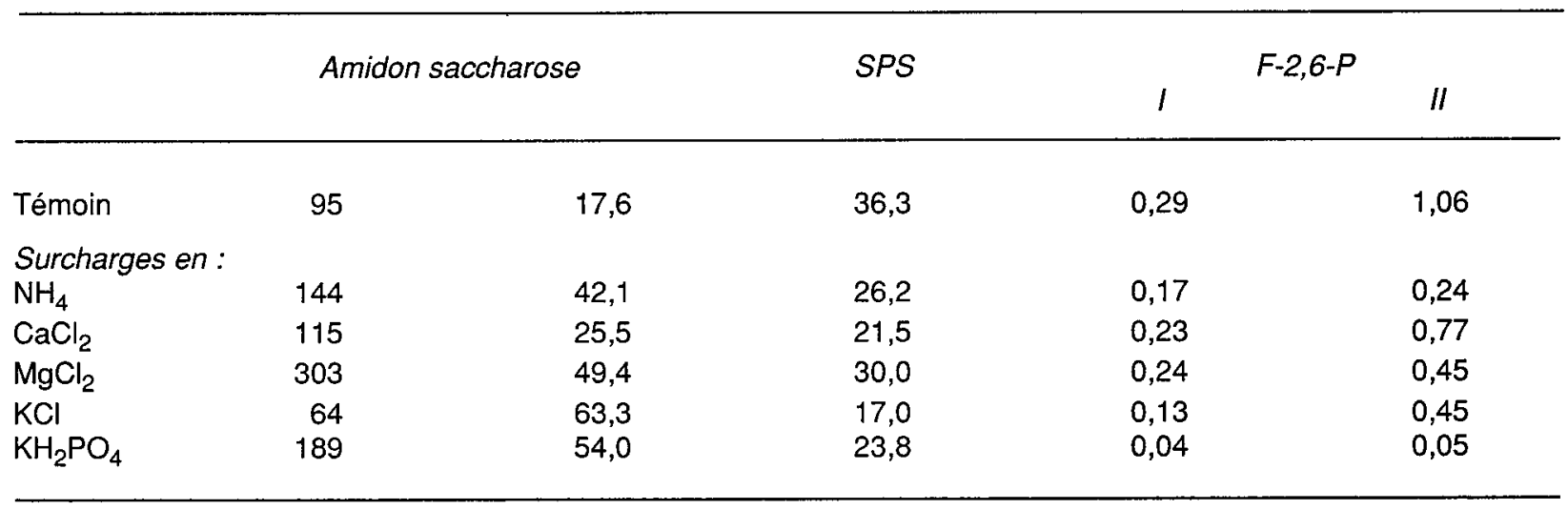

mettent pas de déterminer les quantités synthétisées, du moins pour le saccharose, leurs variations sont un bon indice de l'effet puits.

Lorsqu'on accroît artificiellement l'importance des organes receveurs, la demande en assimilats devient plus grande et davantage de carbone photosynthétique est orienté vers le saccharose. Toutefois, comme ce dernier est rapidement exporté, loin d'observer une augmentation de teneur dans la feuille, on constate une diminution (fig 3). En revanche, l'amidon foliaire est défavorisé et sa teneur diminue. Inversement, lorsqu'on réduit l'appel d'assimilats exercé par les organes receveurs, le saccharose stagne dans la feuille, sa teneur augmente et, par un effet de rétro-inhibition, la synthèse est freinée. II en résulte une orientation plus grande du carbone photosynthétique vers l'amidon dont la teneur foliaire croît.

Ces résultats obtenus sur des plantes entières en période de remplissage des graines sont en accord avec ceux décrits pour des feuilles isolées ou de jeunes pieds de soja (Rufty et Huber, 1983). Ils éclairent le problème de la régulation in situ de la distribution du carbone photosynthétique entre amidon et saccharose. Dans une revue récente, Stitt et Quick (1989) considèrent que les mécanismes primaires de cette régulation se déroulent dans le hyaloplasme où la SPS, la fructose-1,6-bisphosphatase, le F-2,6-P contrôlent d'une manière fine la synthèse du saccharose (Stitt et al, 1984, 1988). L'étude de mutants montre qu'une synthèse plus faible de saccharose s'accompagne généralement d'une formation plus importante d'amidon. Mais la réciproque n'est pas vraie : des mutants pour lesquels la formation d'amidon est ralentie ne pré- sentent pas une synthèse accrue de saccharose. À côté de ces éléments fondamentaux de la régulation, nos résultats mettent en relief le rôle joué par les transferts de photosynthétats vers les organes puits. Lorsque les migrations diminuent (tableau II), le saccharose marqué s'accumule dans la feuille et sa synthèse faiblit; davantage de carbone est alors intégré dans l'amidon.

Pour influer sur les transferts sans avoir à supprimer des organes (sources ou receveurs) et à la suite des travaux de Doman et Geiger (1979) sur l'action des concentrations élevées en $\mathrm{K}^{+}$, des cultures suralimentées en ions minéraux ont été réalisées. Dans tous les cas étudiés (fig 4), les transferts sont nettement ralentis; il en résulte une forte augmentation de la teneur foliaire en saccharose par rapport aux cultures témoins (tableau III). Cette accumulation du diholoside exerce un effet inhibiteur sur sa formation; de plus, la SPS, l'une des enzymes clés de sa synthèse, présente une activité moindre. En même temps, l'amidon devient plus abondant (tableau III). Toutefois, en présence de $\mathrm{CaCl}_{2}$, la teneur foliaire en amidon n'augmente que légèrement; elle diminue même dans le cas de surcharges en $\mathrm{KCl}$. Ceci est à rapprocher des résultats obtenus en conditions de stress hydrique (Bensari et al, 1990) : l'amidon qui s'accumule dans un premier temps est ensuite progressivement hydrolysé, permettant ainsi la survie de la plante. En conséquence, une faible teneur en amidon vers la fin de la journée est lindice de conditions culturales défavorables.

À la différence des autres ions minéraux, le phosphate inorganique et les composés phosphorylés dont il permet la formation participent directement à la voie métabolique conduisant au 
saccharose. D'après les travaux de Stitt et ses collaborateurs, l'abondance du phosphate inorganique limite la synthèse de l'amidon (Heldt et al, 1977 ) et favorise celle du saccharose (Stitt et Schreiber, 1988) malgré une teneur élevée en F2,6-P dont le rôle régulateur sur la fructose-1,6biphosphatase est bien connu (Stitt, 1987). Ces résultats ont été obtenus avec des chloroplastes ou des feuilles isolées au cours des minutes qui suivent la fourniture de phosphate inorganique. Dans notre travail, les conditions de suralimentation sont très différentes : il s'agit de plantes entières qui absorbent le phosphate inorganique par le système racinaire. Elles s'adaptent progressivement et, après $7 \mathrm{j}$, la faible teneur foliaire en F-2,6-P (tableau III) devrait être un élément favorable à la synthèse du saccharose; mais l'abondance du $P_{i}$ inhibe également l'activité de la SPS et réduit, par le fait même, la formation du saccharose. Dans ces conditions, davantage de carbone s'oriente vers l'amidon et les exportations vers les organes receveurs sont diminuées.

D'un point de vue agronomique, les irrigations fertilisantes favorisent les transferts vers les gousses; il en résulte une synthèse accrue de saccharose au détriment de l'amidon. Mais cet effet bénéfique peut être contrarié par des facteurs climatiques. Ainsi l'abaissement de température durant plusieurs nuits consécutives, en période de remplissage des graines, ralentit nettement les migrations; l'hydrolyse nocturne de l'amidon est affectée : elle est d'autant plus limitée que la plante a reçu des irrigations fertilisantes (Calmés et al, 1988). Une feuille qui, en début de journée, contient peu d'amidon et de saccharose témoigne de transferts satisfaisants et correspond à de bonnes conditions de productivité.

\section{RÉFÉRENCES}

Bensari M, Calmès J, Viala G (1988) Photosynthèse et répartition du carbone entre le saccharose et l'amidon, au cours de la photopériode, dans la feuille de soja. CR Séances Acad Sci Paris, sér III 306, 351356

Bensari M, Calmés J, Viala G (1990) Répartition du carbone fixé par photosynthèse entre l'amidon et le saccharose dans la feuille de Soja. Influence d'un déficit hydrique. Plant Physiol Biochem 28, 113-121

Calmés J, Viala G (1987) Relations organes donneurs et organes receveurs chez le soja : migrations vers les graines des composés azotés foliaires. CR Acad Sci Paris, sér III 305, 165-169

Calmés J, Naudy-de Serres M, Viala G, Blanchet R (1988) Influence des basses températures noc- turnes sur les transferts des assimilats foliaires vers les graines de soja. Inf Tech CETIOM 104, 23-28

Carlson DR, Brun WA (1984) Alterations of ${ }^{14} \mathrm{C}$ assimilates partitioning in leaves of soybeans having increased reproduction loads at one node. Plant Physiol 75, 887-890

Davin L (1987) Contribution à l'étude de la saccharose-phosphate synthase de l'orge (Hordeum vulgare $\mathrm{L}$ ) et du soja (Glycine max $\mathrm{L}$ merr). Thèse Doct $3^{\mathrm{e}}$ cycle, univ Paul Sabatier, Toulouse, $97 \mathrm{p}$

Doman DC, Geiger DR (1979) Effect of exogenously supplied foliar potassium on phloem loading in Beta vulgaris L. Plant Physiol 64, 528-533

Fehr WR, Caviness CE, Burnood DT, Pennigton JS (1971) Stage of development description for soybean Glycine max. Crop Sci 11, 929-931

Foyer $\mathrm{CH}$ (1987) The basis for source-sink interaction in leaves. Plant Physiol Biochem 25, 649-657

Foyer $\mathrm{CH}$ (1988) Feedback inhibition of photosynthesis through source-sink. Plant Physiol Biochem 26, 483-492

Gerhardt R, Stitt M, Heldt HW (1987) Subcellular metabolite levels in spinach leaves. Regulation of sucrose synthesis during diurnal alterations in photosynthetic partitioning. Plant Physiol 83, 399-407

Heldt HW, Chon CJ, Maronde D, Herold A, Stankovic ZS, Walker DA, Kraminer A, Kirk MR, Heber U (1977) Role of orthophosphate and other factors in the regulation of starch formation in leaves and isolated chloroplasts. Plant Physiol 59, 1146-1155

Huber SC, Rogers HH, Mowry FL (1984) Effects of water stress on photosynthesis and carbon partitioning in soybean (Glycine max $L$ Merr) plants grown in the field at different $\mathrm{CO}_{2}$ levels. Plant Physiol 76, 244-249

Leegood RC, Labate CA, Huber SC, Neuhaus HE, Stitt M (1988) Phosphate sequestration by glycerol and its effects on photosynthetic carbon assimilation by leaves. Planta 176, 117-126

Rufty TW, Huber SC (1983) Changes in starch formation and activities of sucrose-phosphate synthase and cytoplasmic fructose-1,6-bisphosphatase in response to source-sink alterations. Plant Physiol $72,474-480$

Rufty TW, Huber SC, Volk RJ (1988) Alterations in leaf carbohydrate metabolism in response to nitrogen stress. Plant Physiol 88, 725-730

Salerno GL, Gamundi SS, Pontis HG (1979) A procedure for the assay of sucrose-synthetase and sucrose-phosphate synthase in plant homogenates. Anal Biochem 93, 196-199

Stitt M (1987) Fructose-2,6-bisphosphate and plant carbohydrate metabolism. Plant Physiol 84, 201204

Stitt $M$, Schreiber $U$ (1988) Interaction between sucrose synthesis and $\mathrm{CO}_{2}$ fixation. III. Response of 
biphasic induction kinetic and oscillations to manipulation of the relation between electron transport, Calvin cycle and sucrose synthesis. J Plant Physiol 133, 263-271

Stitt M, Quick WP (1989) Photosynthetic carbon partitioning: its regulation and possibilities for manipulation. Physiol Plant 77, 633-641

Stitt M, Kurzel B, Heldt HW (1984) Control of photosynthetic sucrose synthesis by fructose-2,6bisphosphate. II. Partitioning between sucrose and starch. Plant Physiol 75, 554-560

Stitt M, Gerhardt R, Wilke I, Heldt HW (1987) The contribution of fructose-2,6-bisphosphate to the regulation of sucrose synthesis during photosynthesis. Physiol Plant 69, 377-386
Stitt M, Wilke I, Feil R, Heldt HW (1988) Coarse control of sucrose phosphate synthase in leaves: alterations of the kinetic properties in response to the rate of photosynthesis and the accumulation of sucrose. Plant 174, 217-230

Van Schaftingen E, Lederer B, Bartrons R, Hers HG (1982) A kinetic study of pyrophosphate fructose-6phosphate phosphotransferase from potato tubers. Application to microassay of fructose-2,6bisphosphate. Eur J Biochem 129, 191-195

Zombré G, Naudy-de Serres M, Viala G, Calmés J, Cavalié G (1990) Teneur en fructose-2,6bisphosphate et formation du saccharose dans les feuilles de soja : étude en fonction du stade de développement. CR Séances Acad Sci Paris, sér III $310,253-258$ 